\title{
Microsatellite instability and mutational analysis of transforming growth factor $\beta$ receptor type II gene (TGFBR2) in sporadic ovarian cancer
}

\author{
A J Alvi, J S Rader, M Broggini, F Latif, E R Maher
}

\begin{abstract}
Aims-To investigate the possible role of mutations in the transforming growth factor $\beta$ receptor type II gene (TGFBRII) in ovarian cancer and its relation to microsatellite instability (MSI), 43 sporadic ovarian tumours were analysed for mutations over the entire coding region of the TGFBRII gene.
\end{abstract}

Methods-Mutational analysis was performed using the polymerase chain reaction (PCR), single strand conformation polymorphism (SSCP) gel analysis, and direct sequencing. MSI analysis included both mononucleotide and dinucleotide microsatellite markers used for radiolabelling and gene scanning.

Results-No pathogenic mutations were detected, although sequencing of the polyadenine (poly A) tract in exon 3 using conventional techniques revealed a spurious frameshift mutation that was not present in the same samples analysed using a proofreading Taq polymerase. MSI analysis demonstrated an MSI negative phenotype in 40 of the 43 tumours. None of the three MSI positive tumours demonstrated MSI for mononucleotide markers only.

Section of Medical and Molecular Genetics, Department of

Paediatrics and Child Health, University of Birmingham,

Birmingham B15 2TT, UK

A J Alvi

F Latif

E R Maher

Molecular

Pharmacology Unit, Department of

Oncology, Istituto di

Recerche

Farmacologiche

"Mario Negri", via

Eritrea, 6220157

Milan, Italy

M Broggini

Department of

Obstetrics and

Gynecology,

Washington University

School of Medicine, St

Louis, Missouri 63110,

USA

J S Rader

Correspondence to:

Professor Maher

e.r.maher@bham.ac.uk

Accepted for publication 1 February 2001
Conclusions-These findings suggest that: (1) MSI (both conventional and mononucleotide) is infrequent in ovarian cancer and (2) inactivation of the MSH2, MLH1, and MSH6 mismatch repair genes and TGFBR2 gene mutations do not play a major role in ovarian cancer tumorigenesis. The spurious TGFBR2 frameshift mutations detected by sequencing after conventional PCR underline the importance of confirming putative mutations in repetitive sequences by alternative methods.

(f Clin Pathol: Mol Pathol 2001;54:240-243)

Keywords: transforming growth factor $\beta$ receptor type II; ovarian cancer; mutational analysis; microsatellite instability

Ovarian cancer is the fourth most common cause of death from cancer in Western women. Reliable means of early detection of the disease do not exist and most patients have advanced disease and a poor prognosis when diagnosed. Epidemiological risk factors include early menarche, late menopause, nulliparity, and the use of fertility stimulating drugs. Multiple pregnancies, prolonged breast feeding, and the use of oral contraceptive pills decrease the risk of ovarian cancer. This lowered risk may largely be accounted for by suppression of ovulation. ${ }^{1}$ Relatively little is known about the molecular pathology of ovarian cancer. In many instances, major insights into the pathogenesis of sporadic cancers have been gained from the identification of familial cancer susceptibility genes. The best recognised causes of ovarian cancer susceptibility are germline mutations in the BRCA1 and BRCA2 tumour suppressor genes ${ }^{2}$ and the hereditary non-polyposis colon cancer syndrome (HNPCC) gene.

HNPCC is characterised by a dominantly inherited susceptibility to colorectal cancer, endometrial, and ovarian cancers, and less frequently urothelial and gastric cancers. ${ }^{3} \mathrm{Re}-$ cently, we and others have reported that in female HNPCC gene carriers the risk of endometrial and ovarian cancers exceeds that of colorectal cancer. ${ }^{4}$ Most cases of HNPCC result from germline mutations in mismatch repair (MMR) genes, principally hMSH2 and hMLH1, and less frequently hMSH6. The signature of tumour MMR gene deficiency is the presence of altered sized fragments for simple repeat sequences (for example, microsatellite markers). This "microsatellite instability" (MSI positive) or "RER positive" (replication error) phenotype has been used as a marker to identify cancers with MMR gene inactivation. ${ }^{67} \mathrm{~A}$ variety of microsatellite polymorphisms may be used to detect MSI including mononucleotide, dinucleotide, trinucleotide, and tetranucleotide repeat polymorphisms. Most commonly, dinucleotide repeat polymorphisms are used. However, compared with hMSH2 and hMLH1, hMSH6 deficiency appears preferentially to impair fidelity of replication of mononucleotide repeats. ${ }^{89}$ Somatic MMR gene inactivation occurs in $15-20 \%$ of all colorectal cancers. The association of germline MMR gene mutations with ovarian cancer susceptibility might predict a role for somatic MMR gene inactivation in sporadic ovarian cancer. However, estimates of the frequency of MSI in sporadic ovarian cancer have been very variable (up to 53\%) and usually based on studies with dinucleotide markers only. ${ }^{10-13} \mathrm{We}$ have identified previously a group of colorectal cancers with MSI for mononucleotide but not dinucleotide microsatellite markers, ${ }^{14}$ and demonstrated a germline MSH6 mutation in an isolated case with mononucleotide only MSI. It is not known whether a similar entity of mononucleotide only MSI ovarian cancer occurs. Frameshift mutations in a poly A tract in the transforming growth factor $\beta$ receptor type II gene (TGFBR2) are frequent in MSI 
positive colorectal cancers. Furthermore, microsatellite stable colorectal cancers often contain inactivating TGFBR2 mutations not involving the poly A tract. ${ }^{15} \mathrm{TGF}-\beta$ inhibits the growth of many cell types. ${ }^{16}$ TGF- $\beta$ signalling occurs after the formation of a complex of two receptors, TGF $\beta$ RII with TGF $\beta$ RI. Briefly, the binding of TGF- $\beta$ to its receptor complex initiates signalling via the Smad family of proteins. Mutations in at least two Smad members (Smad 2 and Smad 4) have been implicated in the development of several types of tumour including colorectal, breast, ovarian, and others. ${ }^{17}$ Recently, mutations in the TGFBR1 gene have also been implicated in the development of colorectal cancer. ${ }^{18}$

Recently, Lynch and colleagues ${ }^{19}$ reported that six of 24 sporadic ovarian tumours analysed demonstrated mutations in TGFBR2, suggesting that TGFBR2 might be an important target in both colorectal and ovarian tumorigenesis. To investigate the role of TGFBR2 inactivation in ovarian tumorigenesis we have analysed a series of 43 sporadic ovarian tumours for TGFBR2 mutations and related this to MSI analysis using both dinucleotide and mononucleotide markers.

\section{Materials and methods}

DNA SAMPLES

Forty three tumour and patient matched normal DNA sample pairs and the Ovcar 5 control cell line were obtained from collaborative laboratories at Washington University School of Medicine (St Louis, USA) and Mario Negri Institute in Milan (Italy), respectively. Patient samples comprised a range of tumour types, both benign and malignant (stages I to IV epithelial tumours: 13 serous, eight endometrioid, eight mixed histological, four teratomas, three mucinous, two granulosas, two unknown, one clear cell, one fibroadenoma, and one unclassified sex cord). All tumours were apparently sporadic.

\section{MICROSATELLITE ANALYSIS}

Two sets of polymerase chain reaction (PCR) amplification were set up. The first used two mononucleotide primers: BAT-26, located in the fifth intron of $\mathrm{hMSH} 2$, and BAT-40, located in the second intron of the 3- $\beta$ hydroxysteroid dehydrogenase gene. Each primer was end labelled before PCR using the T4 kinase enzyme and ${ }^{32} \mathrm{P}-\mathrm{ATP}$ as radioactive substrate. PCR amplification using radioactive primers comprised one cycle of five minutes at $95^{\circ} \mathrm{C}$; 35 cycles of one minute each at $95^{\circ} \mathrm{C}$, $56^{\circ} \mathrm{C}$, and $72^{\circ} \mathrm{C}$; and one cycle of five minutes at $72^{\circ} \mathrm{C}$. A standard Taq polymerase was used for this PCR (Gibco/Life Technologies, Paisley, UK). Radioactive PCR products were run on $6 \%$ denaturing polyacrylamide gels (Sequagel-6; National Diagnostics, Hessle, Yorkshire, UK) for 2.5 hours at $50 \mathrm{~V}$, followed by drying of the gels on to Whatman paper, and overnight exposure on to Kodak T-MAT L/RA autoradiography film.

The second PCR used three fluorescently labelled dinucleotide microsatellite markers: D2S123, D8S255, and D13S175 (Operon
Technologies, Crawley, West Sussex, UK). Fluorescent primers were used in a hot start PCR amplification using standard Taq polymerase. Cycling conditions were: five minutes at $95^{\circ} \mathrm{C} ; 25$ cycles of 30 seconds at $95^{\circ} \mathrm{C}$, 30 seconds at $56^{\circ} \mathrm{C}$, and 30 seconds at $72{ }^{\circ} \mathrm{C}$; and one final cycle of five minutes at $72^{\circ} \mathrm{C}$. A $2 \mu 1$ aliquot of each PCR product was mixed with $1 \mu \mathrm{l}$ of deionised formamide and $1 \mu \mathrm{l}$ of analysis mix consisting of $0.75 \mu \mathrm{l}$ of loading buffer (Blue Dextran and EDTA) and $0.25 \mu \mathrm{l}$ GeneScan 350 size standards (Applied Biosystems, Warrington, Cheshire, UK). Each sample was run on polyacrylamide gels using an $\mathrm{ABI}$ 373 automated DNA stretch sequencer. Results were analysed using the GeneScan 2.1 software provided with the sequencer.

MUTATION ANALYSIS OF THE TGFBR2 GENE

For analysis of the entire coding region of the TGFBR2 gene, the seven exons were divided into 10 PCR amplification products (with sizes ranging from 182 to $294 \mathrm{bp}$ ) and were amplified using published primer sequences. ${ }^{20}$ PCR products were first analysed on single strand conformation polymorphism (SSCP) gels. For each tumour, $5 \mu \mathrm{l}$ of PCR sample was mixed with $5 \mu \mathrm{l}$ of loading buffer and denatured at $96^{\circ} \mathrm{C}$ for 10 minutes, then immediately placed on ice and loaded on to an $8 \%$ polyacrylamide gel. Samples were electrophoresed at $110 \mathrm{~V}$ overnight with chilled circulating buffer $(0.5 \times \mathrm{TBE}$ (Tris borate EDTA). Gels were silver stained after 16 hours of electrophoresis. Briefly, gels were fixed for five minutes in $10 \%$ ethanol, oxidised in $1 \%$ nitric acid, rinsed in distilled water, incubated in a $0.1 \%$ silver nitrate solution for 15 minutes, rinsed again in distilled water, then incubated in a $1: 1$ solution of freshly mixed $0.28 \mathrm{M}$ sodium carbonate and $0.019 \%$ formaldehyde to reveal DNA bands. Direct sequencing was performed on three exons (exons 3, 4.3, and 5) using an ABI 377 automated sequencer. For analysis of exon 3, which contains a 10 adenine repeat sequence, 1.25 units of proofreading Taq (Pfu Turbo; Stratagene Europe, Amsterdam, the Netherlands) and its own $10 \times$ buffer was used because it was shown in our early analyses that Taq polymerase lacking proofreading capabilities was subject to slippage in the poly A region and resulted in consistent false frameshift readings. PCR amplification using Pfu Turbo was as follows: one cycle of denaturation at $95^{\circ} \mathrm{C}$ for five minutes; 35 cycles consisting of one minute at $95^{\circ} \mathrm{C}$, one minute at $55^{\circ} \mathrm{C}$, and one minute at $72^{\circ} \mathrm{C}$; followed by one cycle at $72^{\circ} \mathrm{C}$ for five minutes. A positive control was included (the Ovcar 5 cell line which has a $1 \mathrm{bp}$ insertion in the poly A of exon 3) in the sequencing analysis to ensure that no mutations were missed by the direct sequencing method.

\section{Results}

ESTABLISHING THE FREQUENCY OF MSI IN OVARIAN CANCER

To determine the frequency of MSI in our series of 43 ovarian tumours we analysed the 

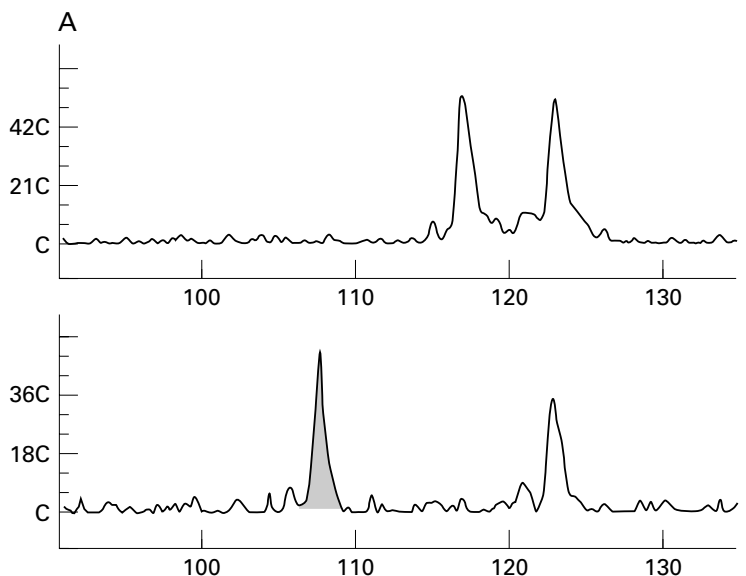

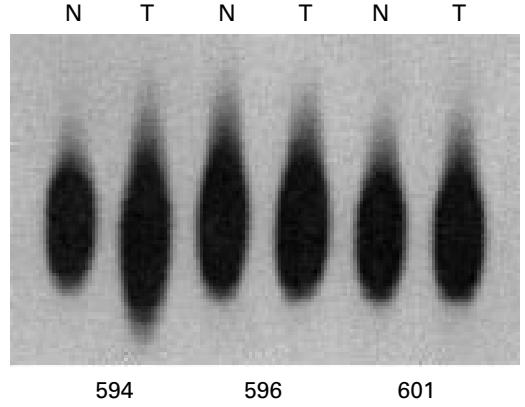

Figure 1 (A) Analysis of dinucleotide instability with fluorescently labelled D13S175 dinucleotide marker in sample 583 showing size instability of D13S175 in the tumour DNA (583T, lower panel) compared with matched normal DNA $(583 N$, upper panel). Allele size in base pairs indicated on the horizontal axes; the units on the vertical axes are arbitrary fluorescent units. (B) Analysis of mononucleotide microsatellite instability (MSI) with the radiolabelled BAT-26 marker demonstrates MSI positivity in sample 594, which shows extra bands in the tumour DNA (T) compared with normal DNA from the same patient (N), and two microsatellite stable samples (596 and 601).

samples using mononucleotide and dinucleotide markers. Conventional criteria for MSI status were used: MSI positive, instability of at least two of five markers; low level MSI, instability at one of five markers; MSI negative, none of five markers showing instability. Forty tumours were MSI negative, and three tumours were MSI positive (instability was observed at both mononucleotides and dinucleotides in two cases) (fig 1). No tumours showed mononucleotide only MSI.

ANALYSIS OF THE TGFBR2 GENE IN OVARIAN

CANCER

To investigate the possible role of TGFBR2 gene mutations in sporadic ovarian cancer, we analysed our samples for mutations in the TGFBR2 gene over its entire coding region. The seven exons of the gene were divided into 10 PCR products (exon 4 is large and was subdivided into four PCR products) and were first analysed by SSCP gel analysis. No abnormal band shifts were detected for any of the exons. In a recent study of 24 sporadic ovarian carcinomas, Lynch et al described mutations in 25\% of their tumour samples in the TGFBR2

A

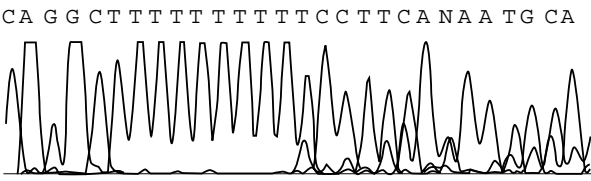

B

CA G G C т т т т т т т т т C C т т NA A tg CA

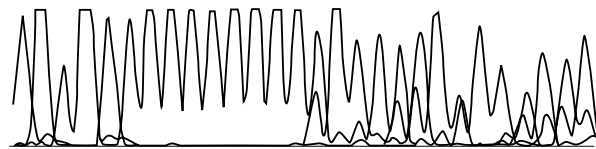

gene. ${ }^{18}$ These mutations included a $1 \mathrm{bp}$ insertion in the poly A of exon 3, resulting in a reading frameshift, and five other nucleotide substitutions resulting in amino acid changes in exons 4.3 and 5 . In addition, the sensitivity of the PCR-SSCP method is less than $100 \%$; therefore, we also looked at these "hotspot" exons in our samples by direct sequencing. When using conventional (non-proofreading) Taq polymerase in the initial PCR step, we consistently noted the presence of "false" frameshifts in the form of a $1 \mathrm{bp}$ deletion in exon 3, which contains a repeated sequence of 10 adenines, for every sample tested including patient tumour and matched normal DNA (fig 2) and unrelated normal control DNA. Therefore, we then used a proofreading Taq polymerase to sequence exon 3 and all false frameshifts previously picked up were no longer detected except for a $1 \mathrm{bp}$ insertion known to be contained within our positive control sample Ovcar 5 (fig 2). No mutations were found in exons 3, 4.3, or 5 for any of the 43 tumour samples either by SSCP or direct sequencing. The positive control sample Ovcar 5 confirmed the validity of the direct sequencing method.

$\mathrm{C}$

T GA A G GAAAAAAAAANCCNGGGGAAANT T

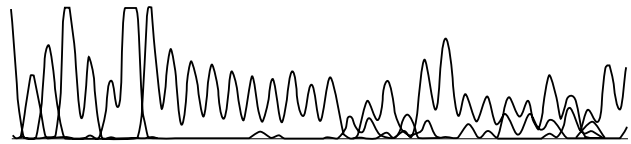

D

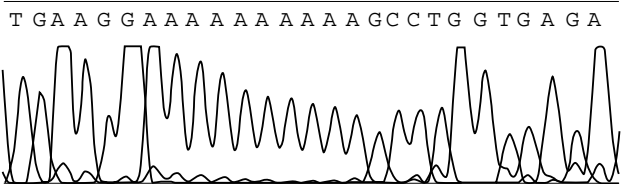

Figure 2 (A) TGFBRII exon 3 sequence analysis of patient matched normal DNA from sample 583 and (B) corresponding tumour DNA. Both normal and tumour DNA display $1 \mathrm{bp}$ deletions in the poly $T$ tract (reverse sequences shown) using conventional Taq polymerase. TGFBRII exon 3 sequence analysis of $(C)$ the Ovcar 5 cell line, which displays a 1 bp insertion in the poly $A$ tract resulting in a frameshift (forward sequence shown) and (D) sample 583 tumour DNA reanalysed using proofreading Taq polymerase showing the absence of a frameshift mutation following the poly $A$ tract. 
These results suggest that TGFBR2 is not a major mutation target in ovarian cancer.

\section{Discussion}

MSI is a hallmark of tumours harbouring mutations in the mismatch repair genes. MSI has been described in many sporadic cancers that can be associated with HNPCC, including colorectal, endometrial, and gastric tumours. Ovarian cancer is a component tumour of some HNPCC families, yet the incidence of MSI has been reported to be much lower than that seen in other sporadic HNPCC associated tumours. ${ }^{10-12}$ Although both we and Allen and colleagues $^{21}$ investigated the possibility that mononucleotide only MSI might be frequent in ovarian cancers, the incidence of MSI in the two studies was very low (three of 43 and three of 26 cases, respectively) and there was no evidence that mononucleotide only MSI is frequent in sporadic ovarian tumours. The number of MSI positive samples in our study (one of eight endometrioid tumours) is too small to be able to correlate MSI with a particular tumour histology or stage. However, Fujita and colleagues ${ }^{11}$ found a significantly higher incidence of dinucleotide MSI in the endometrioid subtype of ovarian cancer (five of 10 tumours) and King and colleagues ${ }^{12}$ found an association between stage I tumours and much higher incidences of MSI (alterations in $75 \%$ of stage I tumours).

Mutations in hMSH6 are preferentially associated with instability at mononucleotide repeats. In females from atypical HNPCC families, a higher frequency $(73 \%)$ of endometrial hyperplastic lesions as well as carcinomas has been associated with germline hMSH6 mutations as compared with carriers of mutations in hMSH2 (29\%) or hMLH1 $(31 \%) .{ }^{22}$ In a previous study, we identified a subgroup of patients with early onset colorectal cancer who displayed mononucleotide only MSI (one of whom was found to have a germline hMSH6 mutation). In our present study, we wanted to determine whether a similar subgroup existed in sporadic ovarian cancer; however, we did not identify a group of ovarian cancers with mononucleotide only MSI, which suggests that hMSH6 mutations are rare in sporadic ovarian cancers. Although TGFBR2 mutations are frequent in sporadic colorectal cancer, TGFBR2 mutations are infrequent in endometrial cancers from patients with germline MSH6 mutations. ${ }^{23}$ This suggests differing mechanisms of tumorigenesis in two HNPCC associated cancers. Our findings also suggest that TGFBR2 is not a major target for mutational inactivation in ovarian cancer. This conclusion differs from that reached by Lynch et al, ${ }^{18}$ who reported that
$25 \%$ of tumour samples contained mutations in this gene. However, another recent study ${ }^{24}$ found that only one of 104 ovarian cancers had mutations in the poly A region of the TGFBR2 gene.

We thank Wellbeing for financial support.

1 Berchuck A, Schildkraut JM, Marks JR, et al. Managing hereditary ovarian cancer risk. Cancer 1999;86:1697-704.

2 Rubin SC, Blackwood MA, Bandera C, et al. BRCA1, BRCA2, and hereditary nonpolyposis colorectal cancer gene mutations in an unselected ovarian cancer population: relationship to family history and implications for genetic testing. Am f Obstet Gynecol 1998;178:670-7.

3 Lynch HT, de la Chapelle A. Genetic susceptibility to non-

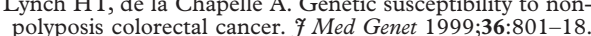
polyposis colorectal cancer. 7 Med Genet 1999;36:801-18.
Dunlop MG, Farrington SM, Carothers AD, et al. Cancer Dunlop MG, Farrington SM, Carothers AD, et al. Cancer
risk associated with germline DNA mismatch repair gene risk associated with germline DNA misma
mutations. Hum Mol Genet 1997;6:105-10.

5 Froggatt NJ, Green J, Brassett C, et al. A common MSH2 mutation in English and North American HNPCC families: origin, phenotypic expression, and sex specific differences in colorectal cancer. f Med Genet 1999;36:97-102.

6 Papadopoulos N, Nicolaides NC, Wei Y-F, et al. Mutation of a mutL homolog in hereditary colon cancer. Science 1994; 263:1625-9.

7 Brassett C, Joyce JA, Froggatt NJ, et al. Microsatellite instability in early onset and familial colorectal cancer. $\mathcal{F} \mathrm{Med}$ Genet 1996;33:981-5.

8 Papadoupoulos N, Nicolaides NC, Liu B, et al. Mutations of GTBP in genetically unstable cells. Science 1995;268:191517.

9 Sia EA, Kokoska RJ, Dominska $M$, et al. Microsatellite instability in yeast: dependence on repeat unit size and DNA mismatch repair genes. Mol Cell Biol 1997;17:28518.

10 Sood AK, Buller RE. Genomic instability in ovarian cancer: a reassessment using an arbitrarily primed polymerase chain reaction. Oncogene 1996;13:2499-504.

11 Fujita M, Enomoto T, Yoshino K, et al. Microsatellite instability and alterations in the hMSH2 gene in human ovarian cancer. Int f Cancer 1995;64:361-6.

12 King BL, Carcangiu M-L, Carter D, et al. Microsatellite instability in ovarian neoplasms. Br f Cancer 1995;72:376-

13 Han H-J, Yanagisawa A, Kato Y, et al. Genetic instability in pancreatic cancer and poorly differentiated type of gastric pancreatic cancer and poorly differe

14 Verma L, Kane MF, Brassett C, et al. Mononucleotide microsatellite instability and germline MSH6 mutation analysis in early onset colorectal cancer. f Med Genet 999;36:678-82

15 Lu S-L, Kawabata M, Imamura T, et al. HNPCC associated with germline mutation in the TGF- $\beta$ type II receptor gene. Nat Genet 1998;19:17-18.

16 Massague J. TGF- $\beta$ signal transduction. Anпи Rev Biochem 1998;67:753-91.

17 Schutte M, Hruban RH, Hedrick L, et al. DCP4 gene in various tumor types. Cancer Res 1996;56:2527-30.

18 Pasche B, Kolachana P, Nafa K, et al. T $\beta \mathrm{R}-\mathrm{I}(6 \mathrm{~A})$ is a candidate tumor susceptibility allele. Cancer Res 1999;59:567882 .

19 Lynch MA, Nakashima R, Song H, et al. Mutational analysis of the transforming growth factor $\beta$ receptor type II gene in human ovarian carcinoma. Cancer Res 1998 58:4227-32.

20 Lu S-L, Zhang W-C, Akiyama Y, et al. Genomic structure of the transforming growth factor $\beta$ type II receptor gene and its mutations in hereditary nonpolyposis colorectal cancers. Cancer Res 1996 56:4595-8.

21 Allen HJ, DiCioccio RA, Hohmann P, et al. Microsatellite instability in ovarian and other pelvic carcinomas. Cancer Genet Cytogenet 2000;117:163-6.

22 Wijnen J, de Leeuw W, Vasen J, et al. Familial endometrial cancer in female carriers of MSH6 germline mutations. Nat Genet 1999;23:142-4.

23 Gurin CC, Federici MG, Kang L, et al. Causes and consequences of microsatellite instability in endometrial carcinoma. Cancer Res 1999;59:462-6.

24 Manderson EN, Mes-Masson A-M, Provencher D, et al. Mutations in a 10-bp polyadenine repeat of transforming growth factor $\beta$-receptor type II gene is an infrequent event in human epithelial ovarian cancer. Clin Genet 2000;57: 151-3. 